\title{
Intracerebral haemorrhage and vasculitis secondary to amphetamine use
}

\author{
V. SALANOVA \\ M.D. \\ R. TAUBNER \\ M.D. \\ Neurology Service, Fitzsimons Army Medical Center Aurora, CO 80045, U.S.A.
}

\begin{abstract}
Summary
We report a case of amphetamine-related intracranial haemorrhage and vasculitis, responding to immunosuppressants. Angiograms obtained before and after therapy are shown; the importance of immunosuppressive therapy is discussed.
\end{abstract}

KEY WORDS: hemiplegia, cyclophosphamide, prednisolone.

\section{Introduction}

Intracerebral haematomas are a rare complication of amphetamine abuse, and may or may not be associated with arteriographic evidence of a vasculitis. We report a further case to draw attention to the important clinical implications of the serious prognosis associated with this disease.

\section{Case report}

An 18-year-old male developed a severe left frontal headache and subsequent progressive right hemiparesis several hours after taking three tablets of amphetamine orally. His past medical history was unremarkable aside from a long history of drug abuse with numerous agents, largely amphetamines and marihuana.

The patient was afebrile with a blood pressure of $106 / 60 \mathrm{mmHg}$ and a pulse rate of 72 per min. He was oriented to person, time and place. Speech, recent and remote memory were normal. He had a dense right hemiplegia and a right hemisensory loss. There was no evidence of trauma or signs of systemic vasculitis.

Investigation revealed a normal blood count, biochemistry, sedimentation rate, platelet count, coagulation studies, antinuclear factor, rheumatoid factor, serum complement levels and blood cultures. The electrocardiogram and chest X-ray were also normal.

The views expressed in this paper are those of the authors and do not express the official views of the Department of the Army or the Department of Defence.
A computed tomographic (CT) head scan (Fig. 1) demonstrated a $2.3 \times 2.7 \mathrm{~cm}$ left intracerebral haematoma, involving the internal capsule and basal ganglia. A $4 \mathrm{~mm}$ left to right shift was noted. Cerebral angiography revealed diffuse bilateral multi-focal areas of ectasia and narrowing in all intracranial arteries, consistent with a vasculitis (Fig. 2). The patient was treated with dexamethasone 24 mg per day intravenously, for 5 days, followed by 50 $\mathrm{mg}$ of prednisone per day. Cyclophosphamide $2 \mathrm{mg}$ per $\mathrm{kg}$ per day was added. He improved steadily walking with assistance 1 week after admission. A repeat angiogram 18 days after the first showed marked improvement (Fig. 3). Complications during the angiography prevented renal and hepatic angiograms from being performed. The prednisone was tapered after 1 month and the patient was discharged 6 weeks after admission with a mild residual right hemiparesis.

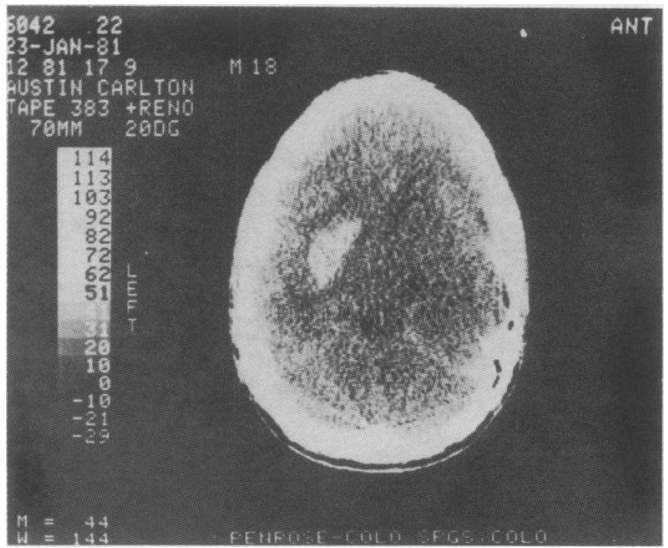

Fig. 1. CT head scan on admission showing a left intracerebral haematoma involving the internal capsule and basal ganglia. 


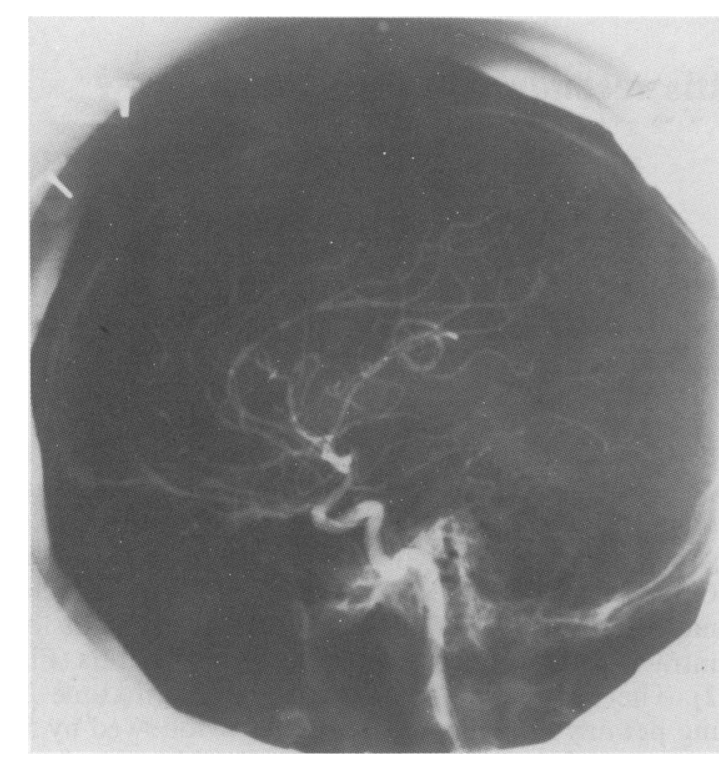

FIG. 2. Cerebral angiogram (lateral view). Showing diffuse multifocal areas of ectasia and narrowing of the anterior and middle cerebral arteries.

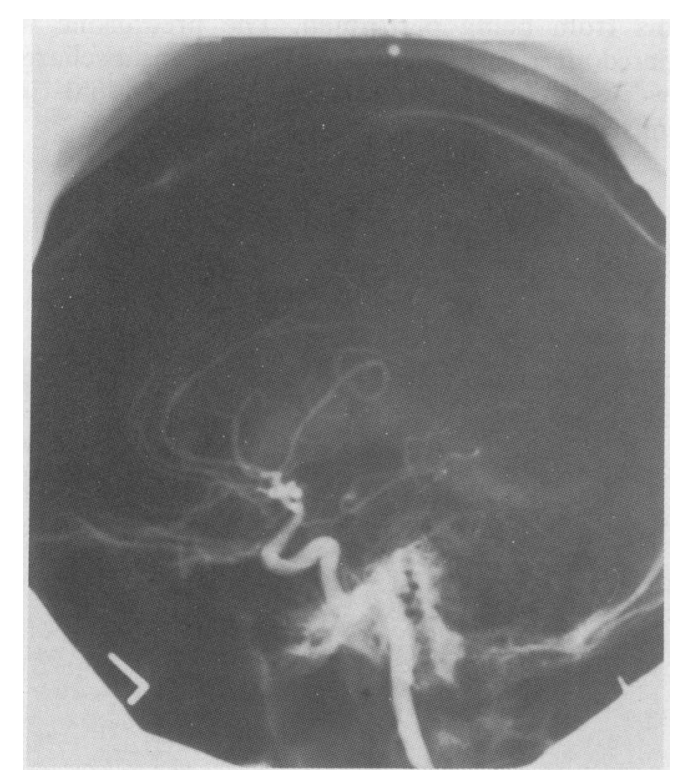

FIG. 3. Cerebral angiogram (lateral view) repeated 18 days after the first one, showing marked improvement.

\section{Discussion}

A small number of patients with intracerebral haemorrhage associated with amphetamines have $\stackrel{\varrho}{\llcorner}$ been reported. Delaney and Estes (1980) reported one case and reviewed 14 others reported in thes literature. Amphetamines were taken intravenouslyo in eight patients and orally in seven. Symptoms 0 consisting of headaches, decrease in the level of consciousness and hemiparesis began minutes after $\overparen{\odot}$ consuming the drug. Five patients were found to have an elevated blood pressure, and in two the $e^{\infty}$ diastolic exceeded $120 \mathrm{mmHg}$. Arteriograms revealed $\vec{O}$ beading of the intracranial arteries in four of the $\overrightarrow{\vec{A}}$ cases studied. In eight cases (five at autopsy) intra- $\omega$ cranial pathological studies were obtained, and in two of these cases an intracranial vasculitis was 3 demonstrated. The findings suggest that the intracer-ebral haemorrhage is due to either a vasculitis or. amphetamine related hypertensive crisis or both.

Amphetamine-related vasculitis has been well defined in the literature, and the pathology of this necrotizing vasculitis angiitis was delineated by을 Citron et al. (1970). The early vascular changes. include fibrinoid angiitis, necrosis of the media andc intima and a leukocytic infiltrate; marked intimal proliferation is seen. In the late stages, muscular and ${ }^{\circledR}$ elastic tissue is replaced by collagen. Medium- anßd $\ddot{\emptyset}$ small-sized arteries and arterioles are involved. The + vessel lumen is usually narrowed considerably in the involved segments and occasionally a nodular ane rysmal dilatation is found, $\mathrm{Yu}$ et al. (1983) reported a patient with amphetamine-related vasculitis and intracerebral haemorrhage, responding to predni- $\mathbb{D}$ sone. Our case similarly responded briskly to immunosuppressive agents, and the angiograms demon- $\frac{0}{3}$ strated the improvement. In view of the five deaths in the 15 patients reviewed by Delaney and Estes (1980), this response certainly underlines the need for urgent immunosuppressive therapy in patients with amphetamine-related vasculitis.

\section{References}

DELANEY, P. \& EsTeS, M. (1980) Intracranial hemorrhage with 0 amphetamine abuse. Neurology, 30, 1125.

Citron, B.P., Halpern, M., MCCarron, M., Lundberg, G.D., MCCORMICK, R., Pincus, I.J., TATTER, D. \& HAVERBACK, B.J. (1970) Necrotizing angiitis associated with drug abuse. New England Journal of Medicine, 283, 1003.

YU, Y.J., COOPER, D.R., Wellenstein, D.E. \& Block, B. (1983) N Cerebral angiitis and intracerebral hemorrhage associated with methamphetamine abuse. Journal of Neurosurgery, 58, 109.

(Accepted 16 November 1983) 